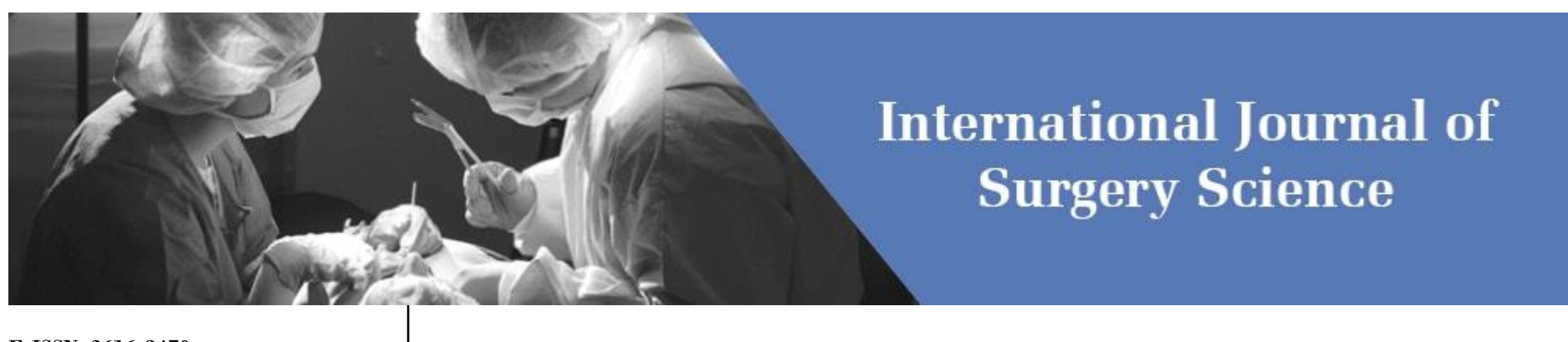

E-ISSN: 2616-3470

P-ISSN: 2616-3462

(C) Surgery Science

www.surgeryscience.com

2021; 5(3): 83-85

Received: 13-05-2021

Accepted: 15-06-2021

Dr. Mehar Chand

M.B.B.S M.S General Surgery,

Civil Hospital Anni, Kullu,

Himachal Pradesh, India

Dr. Kanika Sharma

M.B.B.S M.S OBG, Civil Hospital

Nadaun, Hamirpur, Himachal

Pradesh, India

Dr. Abhey Minhas

M.B.B.S M.S General Surgery,

Civil Hospital Arki, Solan,

Himachal Pradesh, India
Corresponding Author:

Dr. Kanika Sharma

M.B.B.S M.S OBG, Civil Hospital

Nadaun, Hamirpur, Himachal

Pradesh, India

\section{A rare case of isolated splenic tuberculosis}

\author{
Dr. Mehar Chand, Dr. Kanika Sharma and Dr. Abhey Minhas
}

DOI: https://doi.org/10.33545/surgery.2021.v5.i3b.741

\section{Abstract}

Splenic tuberculosis (splenic TB) is extremely rare and has no characteristic symptoms or abnormal imaging findings. Therefore, it is likely to be misdiagnosed as carcinoma of spleen, splenic abscess, lymphoma, rheumatic fever or others. Isolated splenic tuberculosis is rare although secondary involvement in miliary TB is common. The misdiagnosis rate is high if there is no tuberculosis history in other organs. In our case report we are discussing about diagnosis, treatment, and literature review on splenic tuberculosis.

Keywords: spleen, tuberculosis, isolated, splenectomy

\section{Introduction}

- Tuberculosis is one of the top 10 causes of death worldwide.

- In 2018, 10 million people fell ill with TB, and 1.5 million died from the disease.

- India has the world's largest tuberculosis cases which is around $26 \%$ of the world TB cases, followed by China and South Africa ${ }^{[1]}$.

- Tuberculosis is caused by bacteria (Mycobacterium tuberculosis) and about one quarter of the world population has latent TB.

- Extra pulmonary tuberculosis accounts for almost $15 \%$ of all cases of tuberculosis and abdominal tuberculosis comprise a mere $3 \%{ }^{[2]}$.

\section{Case report}

\section{History}

- 44 years old male.

- History of fever, documented up to $103^{\circ} \mathrm{F}$, remittent not associated with chills and rigor

- No H/O pain abdomen

- No H/O weight loss, decreased appetite

- No respiratory, cardiovascular, or genitourinary complaints.

- No H/O previous abdominal surgery or trauma.

- $\quad$ Not a K/C/O TB/DM

\section{Examination}

- Abdomen was soft, no distention.

- No abdominal guarding, rigidity, or rebound tenderness.

- There was no lump palpable and no organomegaly

- Cardiovascular, respiratory, nervous system and musculoskeletal examination were unremarkable.

\section{Investigations}

- USG abdomen: Spleen measuring $12.8 \mathrm{~cm}$ slightly enlarged and showing multiple heterogeneous lesions with maximum size $4.4 \times 4.8 \mathrm{~cm}, \mathrm{~S} / \mathrm{O}$ multiple splenic abscesses.

- CECT abdomen

- Spleen measuring $10.2 \mathrm{~cm}$ in CC extent and showing multiple large peripherally enhancing cystic lesions scattered throughout the spleen with largest measuring $4.7 \mathrm{~cm}$ with CC extent of $6.8 \mathrm{~cm}$. Some of them showing thin internal septations with CT value of $30 \mathrm{HU}$.

- Large multiple lymph nodes in pre/paraaortic, perigastric, perisplenic and peripancreatic region largest measuring $16 \times 9 \mathrm{~mm}$. Some of them showing peripheral enhancement with central hypodensity suggestive of necrosis. 
Blood Investigations: ESR: 100mm at $1 \mathrm{hr}$,

Other blood investigations were WNL

- CXR-PA view: WNL

- USG guided aspiration done

- Pus C/S:- Sterile

- CBNAAT: Not detected

- ZN staining: No organism detected

- GM Staining: No organism detected

\section{Treatment}

Patient was managed conservatively on medical treatment but did not respond so patient was planned for Surgery.

\section{Surgery}

- Elective splenectomy was done

- Operative findings: Multiple whitish nodules were present over surface of spleen.

- Spleen was adherent to left kidney, diaphragm and splenic flexure.

- Spleen dissected, pedicle ligated and cut and splenectomy done

- On cut section: Multiple large yellowish white mass present.

- Imprint Smear from spleen: spleen shows cellular smear revealing lymphoid cells, macrophages, neutrophils, isolated epithelioid change, epithelioid granuloma, amorphous necrotic debris, lymphoglandular bodies and RBCs, S/O tuberculosis

- Patient was put on ATT in post-op period.

\section{Discussion}

\section{Splenic tuberculosis}

- Epidemiological prevalence of splenic tuberculosis is difficult to ascertain as there has been few isolated case reports of the splenic tuberculosis from the different parts of the world. In a large series of 37 cases with focal lesions of the spleen, Joazlina et al. found only 4 cases having the tuberculous etiology ${ }^{[3]}$.

- Splenic tuberculosis usually occurs following the haematogenous spread of infection, as a part of disseminated disease, or, occasionally due to contiguous spread of infection. Immunodeficiency is an important risk factor for splenic tuberculosis ${ }^{[4]}$.

\section{Presentation}

- There are no specific symptoms for establishing the diagnosis of splenic TB ${ }^{[6]}$.

- Splenic enlargement in association with pyrexia of uncertain origin is a clinical sign commonly observed ${ }^{[4]}$.

- The clinical presentation of splenic TB is often nonspecific, making the diagnosis difficult and is, probably, one of the reasons for a lower prevalence. Splenic TB should be considered in patients presenting with fever of undetermined origin and abdominal pain ${ }^{[4]}$.

\section{Investigations}

- Prior to the advent of ultrasonography and computed tomography (CT), it was very diffficult to make the diagnosis. At present, CT is the preferred imaging modality.

- The characteristic CT features of splenic tuberculosis include solitary / multiple nodular or saccular foci or hypodense areas in the spleen ${ }^{[5]}$.

\section{Treatment}

- The first-line management of the splenic tuberculosis is considered to be anti-tubercular chemotherapy with a significant number of the patients responding to it. Surgery may be appropriate in subjects having rupture of the spleen or if the anti-tubercular treatment recurrence ${ }^{[4]}$.

- Treatment for tuberculosis should last for more than 6 months. Standard anti-tuberculosis medication should be taken preoperatively and postoperatively if an operation is carried out ${ }^{[7]}$.
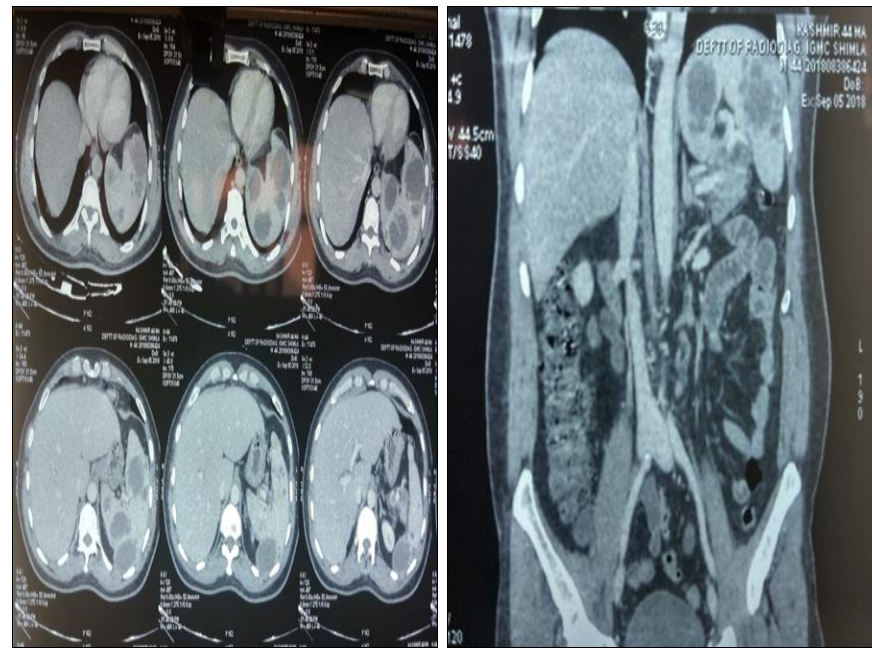

Fig 1: CECT showing multiple cystic lesions in spleen

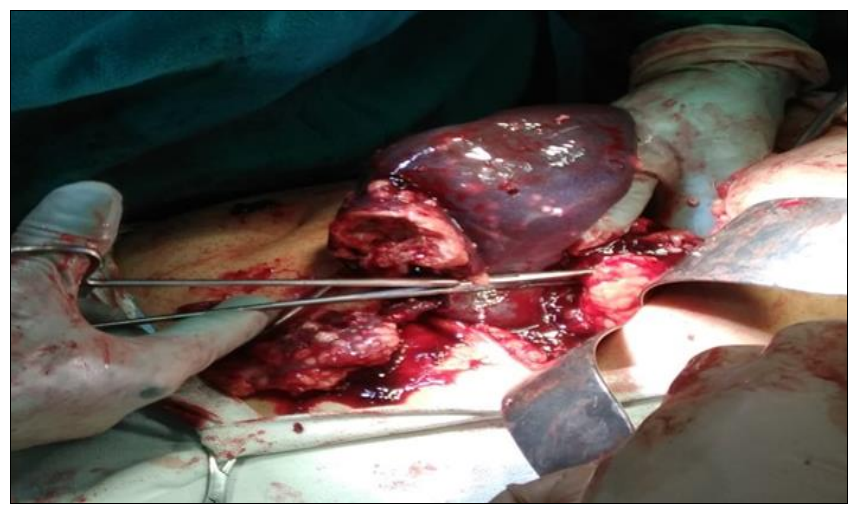

Fig 2: Intra-op picture showing whitish nodules over surface of spleen

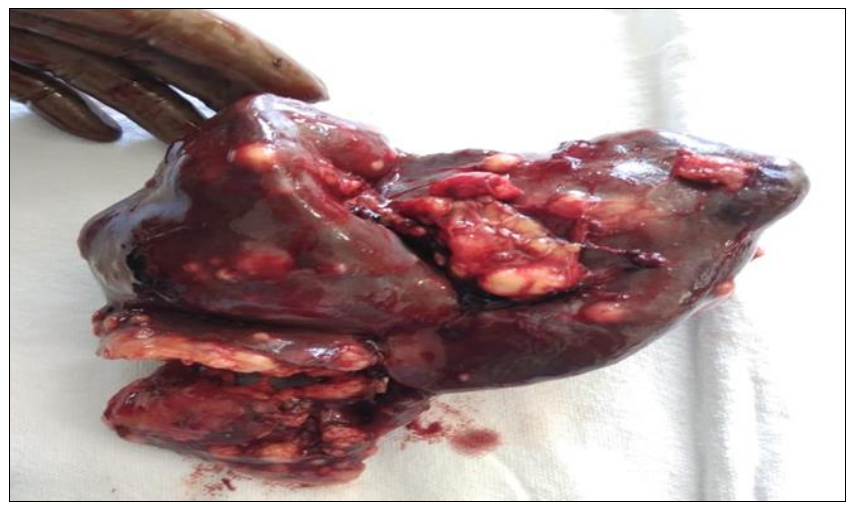

Fig 3: Multiple whitish nodules over spleen 


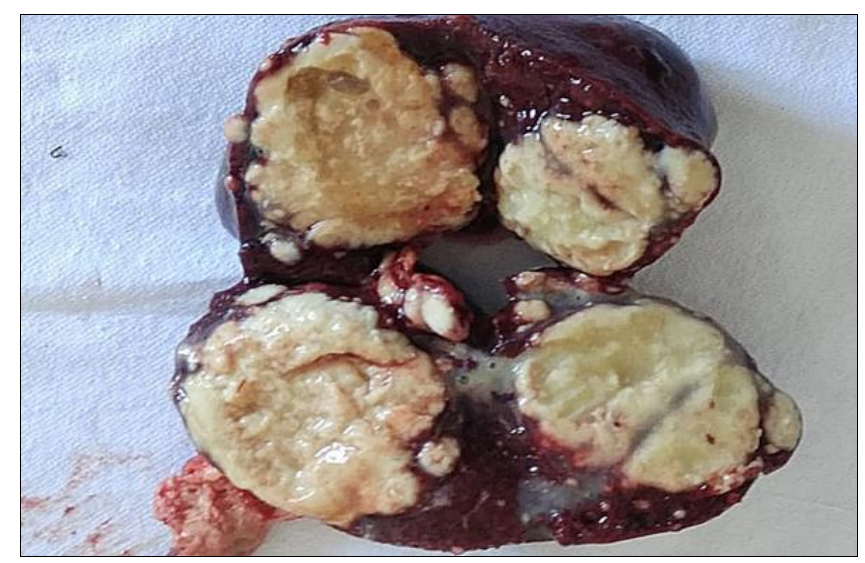

Fig 4: Multiple large yellowish white mass on cut section

\section{References}

1. Rosado E, Penha D, Paixao P, Costa AMD, Amadora PT. Abdominal tuberculosis - Imaging findings. Educational exhibit; ECR 2013, C-0549.

2. Debi U. Abdominal tuberculosis of the gastrointestinal tract: Revisited. World Journal of Gastroenterology 2014;20(40):14831.

3. Joazlina ZY, Wastie ML, Ariffin N. Computed tomography of focal splenic lesions in patients presenting with fever. Singapore Med J 2006;47:37-41.

4. Gupta P, Fotedar S, Agarwal D, Sansanwal P. Tuberculosis of spleen presenting with pyrexia of unknown origin in a non-immunocompromised woman. Lung India 2008;25(1):22.

5. Xie R, Zhou X, Chen J. CT in diagnosis of tuberculosis of liver and spleen. Zhongua Jie $\mathrm{He} \mathrm{He} \mathrm{Hu} \mathrm{Xi} \mathrm{Za} Z \mathrm{Zhi}$ 1999;22:237-38.

6. Ozgüroğlu M, Celik AF, Demir G, Aki H, Demirelli F, Mandel $\mathrm{N}$ et al. Primary splenic tuberculosis in a patient with nasal angiocentric lymphoma: mimicking metastatic tumor on abdominal CT. J Clin Gastroenterol 1999;29:9698.

7. Feng Zhan, Chang-Jun Wang, Ju-Ze Lin, Pei-Jin Zhong, Wei-Zhong Qiu, Hua-Huan Lin et al. World J Gastrointest Pathophysiol 2010;1(3):109-111. Published online 2010Aug 15 . 